\title{
AOR
}

Selected Papers of \#AolR2021:

The 22nd Annual Conference of the Association of Internet Researchers Virtual Event / 13-16 Oct 2021

\section{MISINFORMATION OR ACTIVISM: MAPPING NETWORKED MORAL PANIC THROUGH AN ANALYSIS OF \#SAVETHECHILDREN}

\author{
Rachel Moran \\ University of Washington Information School, Center for an Informed Public \\ Stephen Prochaska \\ University of Washington Information School, Center for an Informed Public \\ Isabelle Schlegel \\ University of Washington \\ Emelia May Hughes \\ University of Washington Information School \\ Owen Prout \\ University of Washington Information School
}

\section{QAnon's co-optation of \#SaveTheChildren-misinformation, activism or both?}

When looking at the recent social-political history of America it is difficult to avoid references to QAnon, a widespread, conservative-leaning conspiracy that amalgamates numerous conspiracies into a single movement. Central to the movement's growth has been a moral panic around child sex trafficking that has served as a gateway for new adherents to become exposed to broader QAnon rhetoric. While such moral panics are certainly not new (Weitzer, 2007), digital social networks have changed the scale and style of conversation, amplifying their effects-as was made clear with the election of a QAnon-supporter to Congress (Rosenberg, 2021) and an insurrection at the U.S. Capitol on January 6th (Rubin et al., 2021). QAnon's co-optation of the \#SaveTheChildren (\#STC) movement exemplifies this networked moral panic and has played a major role in facilitating the spread of misinformation under a banner of moral authority.

The \#STC movement exists as a peculiar mix of conspiracy, misinformation, and disinformation that is often difficult to disentangle from participants' social and political

Suggested Citation (APA): Moran, R., Prochaska, S., Schlegel, I., Hughes, E., Prout, O. (2021, October). Misinformation Or Activism: Mapping Networked Moral Panic Through An Analysis Of \#Savethechildren. Paper presented at AoIR 2021: The 22nd Annual Conference of the Association of Internet Researchers. Virtual Event: AolR. Retrieved from http://spir.aoir.org. 
identities. Accordingly, it is a pertinent case study for understanding both the anatomy and consequences of networked social movements in an era of information disorder (Wardle \& Derakhshan, 2017). This paper thus explores the proponents of \#SaveTheChildren on social media platform Instagram - paying particular attention to their motivations, tactics and desired outcomes-in order to explore how networked social movements build knowledge and organize in spaces that are vulnerable to the spread of misinformation and conspiracy theories. To better understand the movement and its participants, we draw upon theoretical frameworks based in Jost, Ledgerwood, \& Hardin's (2008) conceptions of system justification theory and shared reality theory as well as Lauren Berlant's (2011) conception of cruel optimism (see also: Young, 2021). The paper explores three primary research questions:

RQ1: Who contributed posts to the \#SaveTheChildren movement?

RQ2: What visual and rhetorical tactics are used by \#SaveTheChildren posters?

RQ3: a) What motivations do \#SaveTheChildren posters claim to?

b) What calls to action do they advocate for to achieve their goals?

Research is drawn from an inductive thematic analysis of Instagram posts related to the \#SaveTheChildren movement. Instagram posts from January 2020 through December 2020 were collected using CrowdTangle based on four different hashtags :

\#Save TheChildren, \#SaveOurChildren, \#EndChildTrafficking and \#ChildLivesMatter. The collected dataset includes both content and account-level metadata and associated content. This includes an image of the post itself (unless removed by Instagram), any accompanying captions, hashtags, and engagement data (likes, shares, comments, and views). Additionally, we collected the account handle and number of followers at the time a post was made. The resulting dataset ( $n=1,400$ posts) was cleaned to remove irrelevant posts and other noise. Five researchers undertook several rounds of coding using an iterative process to capture emergent themes and categorical information. Emergent themes included direct QAnon and conspiratorial references (such as "adrenochrome" - a drug QAnon-adherents believe is harvested by elites through human sacrifice), knowledge-building practices (including the sharing of disputed facts and statistics and "friend of a friend" anecdotes), emotional motivations, persuasive techniques (in the main related to "doing your research" and ignoring mainstream media and "Big Tech"), and a variety of calls to action that included both online and offline actions.

An account-level analysis highlights that the primary contributors to the \#SaveTheChildren movement are conservative leaning, white, highly religious femalepresenting accounts with a particular emphasis on motherhood. Additional archetypes included Instagram influencer accounts such as fitness influencers, small business owners, and brands-many of whom did not engage previously with QAnon but became either casual or increasingly vocal proponents of QAnon-inspired conspiracies under the \#SaveTheChildren banner. Further, accounts specifically focused on propagating diverse conspiracy theories were also prevalent within the dataset. 
Content-level analysis highlights the emergence of common stylistic and rhetorical strategies, often associated with the type of account (i.e., conspiracy accounts often relied on different strategies than influencer accounts). Conspiracy accounts relied heavily on memes and highly provocative rhetorical questions aimed at criticizing mainstream media, telling their audience to "wake up" and stop believing mainstream lies. In comparison, feminine/motherhood accounts relied heavily on a pastel visual style and/or pictures of themselves and their children combined with child trafficking statistics intended to shock or outrage their audience. These statistics were often uncited or directly pulled from OUR Rescue, a contentious nonprofit organization (Stackpole, 2015). Alternatively, influencer accounts tailored their strategy to their audience, using numerous hashtags and provocative questions. All account archetypes relied heavily on emotional and moralistic rhetoric, often drawing an implied moral line between those against child trafficking (i.e., themselves) and those not against child trafficking (i.e., anyone who doesn't agree with them).

An analysis of the claimed motivations within the \#SaveTheChildren movement highlights the utility of moral-high ground arguments as a useful trope for advancing other motivations, often individual or political. For the feminine/motherhood account archetype, there was a strong motivation to publicly demonstrate that they are a good, moral person by showing support for an undeniably moral cause connected to their political and religious identity. Alternatively, influencer accounts seemed to utilize the movement to increase their follower account or business, often directly asking for follows or selling merchandise. Conspiracy accounts seemed wholly motivated by spreading their worldview, often using rhetoric that connected the \#SaveTheChildren movement with specific QAnon conspiracy theories. An overarching commitment to "ending" trafficking or "saving" children thus emerged as a uniting theme, bringing together differing communities with different underlying or secondary motivations for joining the movement.

Posters almost always included calls to action within their posts, although those same calls often reflected a lack of understanding of what trafficking actually looks like. The most common call to action was to "speak out" —often with the implication that child trafficking is not talked about by mainstream media. Further, calls to concrete and offline actions were rare, with posters instead seeing "success" for the movement in terms of individualized notions of visibility and collective awareness with no real contemplation of how this may be capitalized on to achieve the abolition of trafficking.

Taken together, the paper illuminates how the \#SaveTheChildren movement is defined more as an identity based, social-political movement rooted in moral panic - rather than one focused on truly supporting human rights. Our analysis highlights how networked moral panics - facilitated by the affordances and tactics of social media — can bring together disparate communities to spread misinformation under the guise of activism. Accordingly, the paper contributes to understandings of the growing attraction of conspiracy theories, the role of social media platforms in amplifying the spread of misinformation and knowledge of the real-world consequences of information disorder. 


\section{References}

Berlant, L. G. (2011). Cruel optimism (Vol. 226). Durham, NC: Duke University Press.

Jost, J., Ledgerwood, A., \& Hardin, CD. (2008). Shared reality, system justification, and the relational basis of ideological beliefs. Social and Personality Psychology Compass, 2, 171-186.

Rosenberg, M. (2020, November 3rd) A QAnon Supporter is Headed to Congress. The New York Times. Retrieved online:

https://www.nytimes.com/2020/11/03/us/politics/qanon-candidates-marjorietaylor-greene.html

Rubin, O., Bruggeman, L., \& Steakin, W. (2021, January 19th) QAnon Emerges as Recurring Theme of Criminal Cases Tied to US Capitol Siege. ABC News. Retrieved online: https://abcnews.go.com/US/qanon-emerges-recurring-themecriminal-cases-tied-us/story?id $=75347445$

Stackpole, T. (2015) The New Abolitionists. Foreign Policy. Retrieved online: https://foreignpolicy.com/2015/07/22/the-new-abolitionists-mexico-dominicanrepublic-human-trafficking-mormon-our/

Wardle, C., \& Derakhshan, H. (2017). Information disorder: Toward an interdisciplinary framework for research and policy making. Council of Europe report, 27, 1-107.

Weitzer, R. (2007). The social construction of sex trafficking: Ideology and institutionalization of a moral crusade. Politics \& Society, 35(3), 447-475.

Young, J. C. (2021). Disinformation as the weaponization of cruel optimism: A critical intervention in misinformation studies. Emotion, Space and Society, 38, 100757. 